\title{
Effectiveness of Acellular Pertussis Vaccine in Older Adults: Nested Matched Case-control Study
}

\author{
Bette C. Liu, ${ }^{1, \oplus}$ Wen-Qiang He, ${ }^{1}$ Anthony T. Newall, ${ }^{1}$ Helen E. Quinn, ${ }^{2,3}$ Mark Bartlett, ${ }^{4}$ Andrew Hayen, ${ }^{5}$ Vicky Sheppeard, ${ }^{6}$ Nectarios Rose, \\ C Raina MacIntyre, ${ }^{7}$ and Peter McIntyre ${ }^{2,8}$
}

${ }^{1}$ School of Public Health and Community Medicine, University of New South Wales, Sydney, Australia; ${ }^{2}$ National Centre for Immunisation Research and Surveillance and University of Sydney, Australia; ${ }^{3}$ Discipline of Child and Adolescent Health, Faculty of Medicine and Health, University of Sydney, Sydney, New South Wales, Australia; ${ }^{4}$ Sax Institute, Sydney, Australia; ${ }^{5}$ University of Technology, Sydney, Australia; ${ }^{6}$ Health Protection, New South Wales Ministry of Health, Sydney, New South Wales, Australia; ${ }^{7}$ Kirby Institute, University of New South Wales, Sydney New South Wales, Australia; and ${ }^{8}$ Department of Women's and Children's Health, University of Otago, Dunedin, New Zealand

Background. Despite recommendations that older adults receive acellular pertussis vaccines, data on direct effectiveness in adults aged over 50 years are sparse.

Methods. A case-control study nested within an adult cohort. Cases were identified from linked pertussis notifications and each matched to 3 controls on age, sex, and cohort recruitment date. Cases and controls were invited to complete a questionnaire, with verification of vaccination status by their primary care provider. Vaccine effectiveness (VE) was estimated by conditional logistic regression, with adjustment for reported contact with children and area of residence.

Results. Of 1112 notified cases in the cohort, we had complete data for 333 cases and 506 controls. Among 172 PCR-diagnosed cases (mean age, 61 years), $11.2 \%$ versus $19.5 \%$ of controls had provider-verified pertussis vaccination, on average, 3.2 years earlier. Adjusted VE against PCR-diagnosed pertussis was 52\% (95\% CI, 15-73\%), nonsignificantly higher if vaccinated within 2 years (63\%; $-5-87 \%)$. Adjusted VE was similar in adults born before 1950, presumed primed by natural infection $(51 \% ;-8-77 \%)$ versus those born 1950 or later who may have received whole-cell pertussis vaccine $(53 \% ;-11-80 \%)(P$-heterogeneity $=0.9)$. Among 156 cases identified by single-point serology, adjusted VE was $-55 \%(-177-13 \%)$.

Conclusions. We found modest protection against PCR-confirmed pertussis among older adults (mean age, 61 years; range, 46-81 years) within 5 years after acellular vaccine. The most likely explanation for the markedly divergent VE estimate from cases identified by single-titer serology is misclassification arising from limited diagnostic specificity in our setting.

Keywords. pertussis; whooping cough; vaccine effectiveness; adult.

Despite widespread vaccination in high-income countries, pertussis remains one of the most poorly controlled vaccine-preventable diseases, with outbreaks continuing to occur every few years [1-3]. Although severe morbidity is highest in the youngest, adults can suffer serious complications [4, 5], with reports suggesting 5-12\% of those aged over 65 with pertussis are hospitalized $[4,6,7]$ and about $8 \%$ develop radiologically confirmed pneumonia [7].

Formulations of acellular pertussis vaccine suitable for adults with reduced amounts of diphtheria and tetanus toxoid (Tdap) have been available since 2000. The only randomized controlled trial in adults reported vaccine efficacy of $92 \%$ (95\% confidence interval [CI], 32-99\%) but was based on 10 cases and excluded those aged more than 65 years [8]. More recent studies reported satisfactory immunogenicity of Tdap in adults aged more than 55 [9] and 65 [10] years; however, data on the effectiveness of

Received 4 June 2019; editorial decision 12 July 2019; accepted 19 August 2019; published online August 26, 2019.

Correspondence: B. C. Liu, School of Public Health and Community Medicine, Samuels Building, UNSW Kensington, NSW 2052, Australia (bette.liu@unsw.edu.au).

Clinical Infectious Diseases ${ }^{\circledR} \quad$ 2019;XX(XX):1-11

(C) The Author(s) 2019. Published by Oxford University Press for the Infectious Diseases Society of America. All rights reserved. For permissions, e-mail: journals.permissions@oup.com. DOI: 10.1093/cid/ciz821 acellular pertussis vaccine in older adults are limited to a single case-control study that reported low but imprecise vaccine effectiveness (VE) (24\%; 95\% CI, $-59-64 \%)$ in a subgroup of adults (mean age, 69 years) [11]. Waning effectiveness of acellular vaccine has been demonstrated in children primed with acellular vaccines [12], but no data are available for adults primed with whole-cell vaccine and/or prior infection.

From 2009 to 2012, the largest Australian state, New South Wales (NSW; population 7 million), implemented a cocooning vaccination program, which provided free Tdap vaccine to adults caring for infants, with vaccine coverage of about $20 \%$ in a cohort of older adults [13]. We conducted a case-control study nested within this cohort, which, based on coverage and expected numbers of pertussis cases, had $90 \%$ power to estimate a minimum VE of $40 \%$, with an $\alpha=.05$. Although recommendations to vaccinate older adults have been in place for some years $[3,14]$, this study is the first to specifically evaluate the magnitude and duration of direct protection in older adults.

\section{METHODS}

We used an established cohort, the Sax Institute's 45 and Up Study [15]. Briefly, between 2006 and 2008, adults aged 45 years 
and over residing in NSW were invited from Australia's national health insurance database, the Department of Human Services (formerly Medicare Australia) enrollment database, to participate. There was oversampling of those living in rural regions and those aged more than 80 years. Overall, 267153 participants (average age, 62 years) completed a questionnaire and consented to be followed up through surveys and linkage to health records. The cohort represented $10 \%$ of the eligible population in the study age range resident in NSW.

For this nested case-control study, in 2016 the cohort was linked to pertussis notifications, hospitalizations, and death records by the NSW Centre for Health Record Linkage [16]. Pertussis notifications are recorded in the NSW Notifiable Conditions Information Management System (NCIMS), which is a statutory database of all notifiable conditions required to be reported by health practitioners and laboratories under the NSW Public Health Act [17]. Cases of pertussis that meet case definitions [18] are recorded. Confirmed cases had either definitive laboratory evidence \{culture or nucleic acid testing (polymerase chain reaction $[\mathrm{PCR}]$ ) or seroconversion in the absence of recent vaccination $\}$ or both a clinical history consistent with pertussis and suggestive laboratory evidence (single high titer of immunoglobulin [Ig] A or IgG antibody to Bordetella pertussis or significant change in IgA or IgG, in the absence of vaccination) (see Appendix) [18]. Thus, cases notified by laboratories based on high single-point serology require active clinical follow-up to be considered confirmed, but not if pertussis is detected by PCR, which is considered definitive. Data in the NCIMS include the notified condition, date of onset, and test type. The NSW Admitted Patient Data Collection records all inpatient hospital admissions in NSW. For each hospitalization the primary diagnosis is coded to the International Classification of Diseases, 10th revision, Australian Modification (ICD-10-AM) [19]; data also include the admission date. Death data are available from the NSW Registry of Births, Deaths, and Marriages and include the date of death. All databases include identifying details enabling linkage to the 45 and Up Study cohort, and at the time of linkage complete data were available until 31 December 2015.

Prior to case and control selection, we excluded cohort participants with a linked record of pertussis (either a notification or a hospitalization where the primary diagnosis was coded as ICD-10-AM A37 Whooping cough) prior to recruitment. We also excluded any participants who linked to a death record.

Cases were defined as cohort participants who, following cohort recruitment, had a first linked record of pertussis (either statutory notification of confirmed pertussis or a hospitalization with a primary ICD-10-AM diagnosis code on admission of A37 Whooping cough). For each case, up to 3 matched controls were selected from the cohort. Controls were matched to cases on age (in 5-year age groups from 45 years), sex, and date of cohort recruitment (4 intervals from before 1 July 2007, 1 July-31 December 2007, 1 January-30 June 2008, after 30
June 2008) and assigned an index date equivalent to the pertussis onset date for their matched case. We invited all cases and matched controls by post and included a questionnaire asking about their vaccination status, their contact with children in the 3 weeks prior to illness or index date, and their healthcare provider details to verify their vaccination status. We also asked cases to provide information about the impact of their pertussis diagnosis on their health and work. For those agreeing to participate we contacted their healthcare provider to verify their pertussis vaccination status. The 45 and Up Study received ethics approval from the University of New South Wales' human research ethics committee (HREC) and this specific study received approval from the NSW Population and Health Services HREC (HREC/10/CIPHS/97). All study participants provided written consent to be included.

\section{Statistical Analysis}

For our main prespecified analyses, we included only respondent cases and their matched controls, for whom we had validated vaccination status from their healthcare provider. Participants were classified as vaccinated if they had a healthcare provider-verified pertussis vaccine administered at least 1 month prior [20] to the pertussis notification or index date (for controls). We estimated the odds ratio (OR) of pertussis infection using conditional logistic regression and examined the effect of adjusting for area of residence, educational level, household income, smoking status, asthma, body mass index (BMI), attending routine cancer screening, and contact with children based on questionnaire responses. Where adjustment variables had missing data, in analyses we included those with missing data in a separate category. We calculated pertussis VE as $(1$-adjusted OR) $\times 100 \%$. We estimated VE overall and by the pertussis identification method (PCR, serology). For PCRconfirmed pertussis cases we estimated VE according to time since vaccine receipt $(<2,2$ to $<5,5+$ years compared with no vaccination) and by age ( $<65$ vs $\geq 65$ years).

To maximize our analysis population we conducted additional analyses. For PCR-confirmed cases who had validated vaccination records but no respondent matched control with a validated vaccination record and who were therefore excluded from our main analysis, we selected new matched controls (on age group, sex, cohort recruitment date) from the pool of controls with a validated vaccination record but who had no respondent matching case with a validated vaccination record.

All analyses were conducted using R version 3.5.1 [21].

\section{RESULTS}

We identified and invited 1112 confirmed cases: 527 (47.4\%) were based on PCR, 566 (50.9\%) on single-titer serology, whereas 19 had no laboratory diagnostic information. We invited 3291 corresponding matched controls. Of those invited, $60 \%$ (671 of 1112 ) of cases and 49\% (1610 of 3291) of controls

2 - CID 2019:XX (XX XXXX) • Liu et al 
agreed to participate, and in participants we obtained validated vaccination status for $69 \%$ (463 of 671) of cases and 67\% (1080 of 1610) of controls (Figure 1).

Compared with nonparticipating cases, participants were more likely to be younger, women, and have higher incomes and educational levels and less likely to be current smokers. Such differences were less apparent for controls (Table A1). Of participants, the proportion of their health practitioners who also responded did not vary substantially by a range of characteristics, although practitioner response rates for cases were slightly greater for older participants and for never smokers (Table A2).

In analyses restricted to cases and their corresponding controls with validated vaccination data (prespecified analysis population), we had 333 cases and 506 controls (Figure 1); 130 cases with validated vaccination status lacked a matched control with

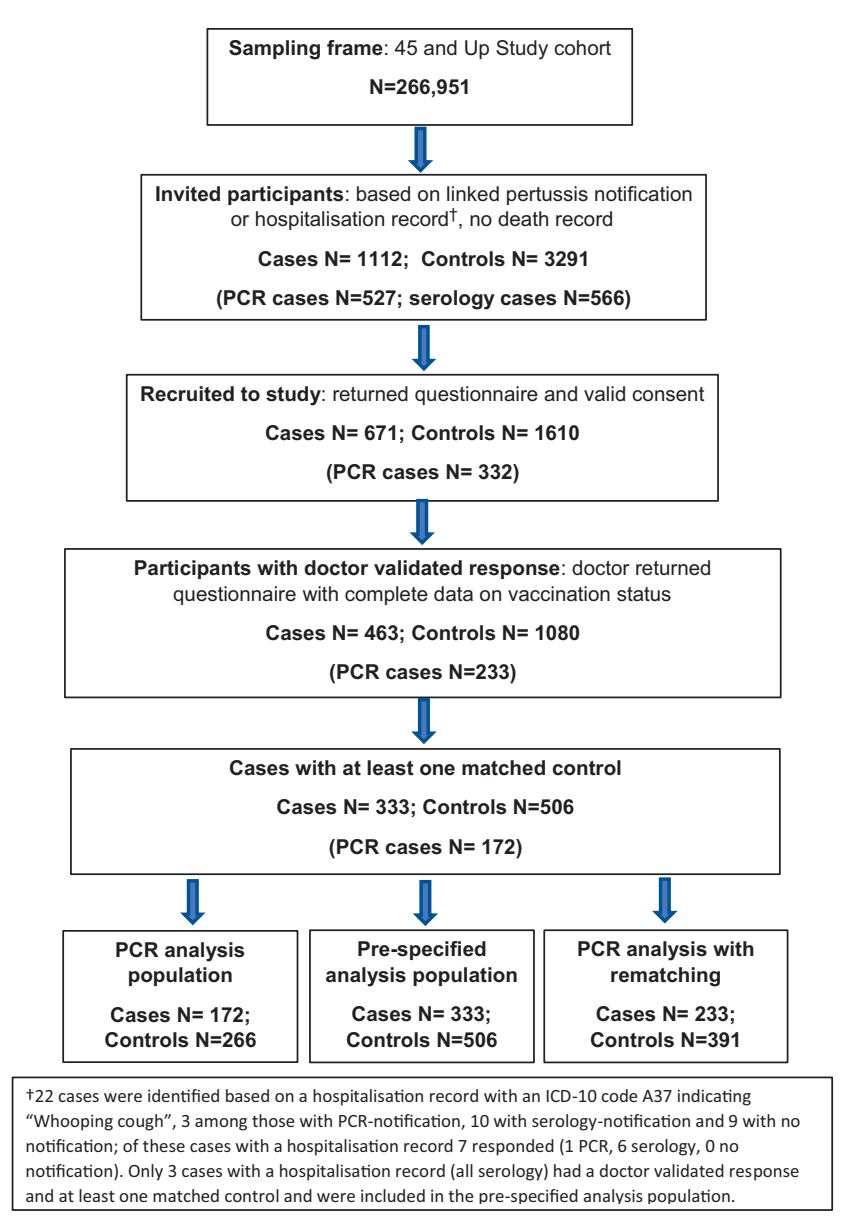

Figure 1. Study sampling frame, recruitment, and analysis population: flow chart. ${ }^{\dagger} 22$ cases were identified based on a hospitalization record with an ICD-10 code A37 indicating "Whooping cough": 3 among those with polymerase chain reaction (PCR) notification, 10 with serology notification, and 9 with no notification; of these cases with a hospitalization record, 7 responded (1 PCR, 6 serology, 0 no notification). Only 3 cases with a hospitalization record (all serology) had a doctor-validated response and at least 1 matched control and were included in the prespecified analysis population. Abbreviations: ICD-10, International Classification of Diseases, 10th revision; PCR, polymerase chain reaction. validated vaccination status. The mean age of participants at diagnosis/index date was 62 years. Characteristics of the included cases and controls were similar except for the proportion reporting contact with children in the 3 weeks prior to the onset/ index date ( $36 \%$ of cases vs $23 \%$ of controls; see Table 1 ). In analyses confined to the subset of cases with a PCR pertussis diagnosis (and their matching controls) we had 172 cases and 266 controls, with a mean age at diagnosis/index date of 61 years (range, 46-81 years). Similar to the prespecified analysis group, the only significant difference between cases and controls was a higher proportion of cases reporting contact with children (Table 2).

In prespecified analyses, the proportions of cases and controls vaccinated were, respectively, 17\% (53 of 333) and 16\% (82 of 506); the average time between vaccination and diagnosis/ index date overall was 3.0 years (range, $0.8-8.8$ years) and for cases and controls, respectively, was 3.0 and 2.9 years. For the PCR-only analysis, the proportions of cases and controls vaccinated were $12 \%$ (20 of 172) and 20\% (52 of 266), respectively, and the mean time since vaccination was 3.2 years. In the prespecified population (333 cases, 506 controls) there was no evidence of VE (adjusted VE, 8\%; 95\% CI, -36-37\%) (see Figure 2). However, VE estimates differed substantially by diagnostic method. Among the PCR-only population, adjusted VE was $52 \%$ (95\% CI, 15-73\%; $P=.01$ ), whereas in the serologyonly population, adjusted VE was $-55 \%$ (95\% CI, $-177-13 \%$; $P=.14 ; P$-heterogeneity $=.005)$. In addition to matching on age, sex, and recruitment date, in the final models the ORs (and VE estimates) were adjusted for reported contact with children and region of residence. Adjustment for education, income, smoking, asthma, BMI, and routine cancer screening did not materially change the estimates and were therefore not included in the final model.

Among the PCR-only population $(\mathrm{N}=438)$, Figure 3 shows $\mathrm{VE}$ overall and by time since vaccination and age group. We found no trend towards declining VE with greater time since vaccination in the PCR-only population, although the category " $\geq 5$ years since vaccination" had a narrow range (mean, 5.9 years; range, 5.0-6.7 years). When stratified by age, VE in those aged younger than 65 years (mean age, 57.6 years) was similar to those aged 65 and over (mean age, 70.0 years) (55\% [95\% CI, 7-78\%] vs 49\% [95\% CI, -32-80\%]; P-heterogeneity $=0.8$ ). As a post hoc analysis we also estimated VE by birth year $(<1950 / 1950+)$ to correspond to prevaccine and whole-cell pertussis vaccine eras [11]. We found no difference in VE: those with birth year before 1950 (mean age, 67.5 years; case $\mathrm{N}=77$ ) had a VE of $51 \%(95 \% \mathrm{CI},-8-77 \%)$ and those born in 1950 or later (mean age, 55.8 years; case $\mathrm{N}=85)$ had a VE of $53 \%(95 \%$ $\mathrm{CI},-11-80 \%$; $P$-heterogeneity $=0.9$ ).

There were 61 PCR cases who had no matched controls with validated vaccination data and were excluded from the main analysis reported in Figure 3. When we included alternate 
Table 1. Characteristics of Cases and Controls in the Prespecified Analysis Population

\begin{tabular}{|c|c|c|c|}
\hline & $\begin{array}{l}\text { Cases, } \\
\mathrm{N}(\%)\end{array}$ & $\begin{array}{l}\text { Controls, } \\
\text { N (\%) }\end{array}$ & $P$ \\
\hline Total & $333(100.0)$ & $506(100.0)$ & \\
\hline \multicolumn{4}{|l|}{ Recruitment date } \\
\hline Before 1 July 2007 & $64(19.2)$ & $92(18.2)$ & .97 \\
\hline 1 July-31 December 2007 & $20(6.0)$ & $29(5.7)$ & \\
\hline 1 January-30 June 2008 & $87(26.1)$ & 131 (25.9) & \\
\hline After 30 June 2008 & 162 (48.6) & $254(50.2)$ & \\
\hline Mean (SD) age at diagnosis/index, years & $61.8(8.0)$ & $61.7(7.8)$ & .85 \\
\hline \multicolumn{4}{|l|}{ Age at diagnosis/index date (years) } \\
\hline $45-49$ & $18(5.4)$ & $33(6.5)$ & .66 \\
\hline $50-54$ & 63 (18.9) & $81(16.0)$ & \\
\hline $55-59$ & 63 (18.9) & 95 (18.8) & \\
\hline $60-64$ & $74(22.2)$ & $134(26.5)$ & \\
\hline $65-69$ & $58(17.4)$ & $85(16.8)$ & \\
\hline$\geq 70$ & $57(17.1)$ & $78(15.4)$ & \\
\hline \multicolumn{4}{|l|}{ Sex } \\
\hline Male & $106(31.8)$ & $156(30.8)$ & .76 \\
\hline Female & $227(68.2)$ & $350(69.2)$ & \\
\hline \multicolumn{4}{|l|}{ Body mass index $\left(\mathrm{kg} / \mathrm{m}^{2}\right)$} \\
\hline$<25$ & 103 (30.9) & $178(35.2)$ & .64 \\
\hline $25-30$ & $124(37.2)$ & $179(35.4)$ & \\
\hline$\geq 30$ & $70(21.0)$ & $100(19.8)$ & \\
\hline Unknown & $36(10.8)$ & $49(9.7)$ & \\
\hline \multicolumn{4}{|l|}{ Highest educational level } \\
\hline Certificate or lower & $229(68.8)$ & $326(64.4)$ & .22 \\
\hline University or higher & $88(26.4)$ & $161(31.8)$ & \\
\hline Unknown & $16(4.8)$ & 19 (3.8) & \\
\hline \multicolumn{4}{|l|}{ Residence } \\
\hline Major city & $150(45.0)$ & $256(50.6)$ & .39 \\
\hline Inner regional & 131 (39.3) & $174(34.4)$ & \\
\hline $\begin{array}{l}\text { Outer regional or } \\
\text { remote or very remote }\end{array}$ & $38(11.4)$ & $59(11.7)$ & \\
\hline Unknown & $14(4.2)$ & $17(3.4)$ & \\
\hline \multicolumn{4}{|l|}{$\begin{array}{l}\text { Annual household income } \\
\text { (AUD) }\end{array}$} \\
\hline$<70000$ & 165 (49.5) & $238(47.0)$ & .77 \\
\hline$\geq 70000$ & $145(43.5)$ & $230(45.5)$ & \\
\hline Unknown & $23(6.9)$ & $38(7.5)$ & \\
\hline \multicolumn{4}{|l|}{ Smoking status } \\
\hline Never & $208(62.5)$ & $304(60.1)$ & .16 \\
\hline Past & $98(29.4)$ & $152(30.0)$ & \\
\hline Current & 11 (3.3) & $33(6.5)$ & \\
\hline Unknown & $16(4.8)$ & $17(3.4)$ & \\
\hline \multicolumn{4}{|l|}{ Asthma } \\
\hline No & $264(79.3)$ & $422(83.4)$ & .32 \\
\hline Yes & $55(16.5)$ & 67 (13.2) & \\
\hline Unknown & $14(4.2)$ & $17(3.4)$ & \\
\hline \multicolumn{4}{|l|}{ Contact with children } \\
\hline No & $176(52.9)$ & $246(48.6)$ & $<.001$ \\
\hline Yes & $119(35.7)$ & $115(22.7)$ & \\
\hline Unknown & $38(11.4)$ & $145(28.7)$ & \\
\hline \multicolumn{4}{|l|}{$\begin{array}{l}\text { Attended routine cancer } \\
\text { screening program }\end{array}$} \\
\hline No & $38(11.4)$ & $51(10.1)$ & .51 \\
\hline Yes & 277 (83.2) & 432 (85.4) & \\
\hline Unknown & $18(5.4)$ & $23(4.5)$ & \\
\hline
\end{tabular}

Percentages are column percentages. Abbreviation: AUD, Australian dollars. matched controls with validated vaccination data but lacking a matched case with validated vaccination data, the number of PCR cases increased to 233 with 391 controls. Adjusted VE did not change significantly (40\%; 95\% CI, 4-63\%) nor did it differ by time since vaccination or by age group (Appendix Figure 1).

For cases notified based on single-point serology and their matched controls $(\mathrm{N}=388)$ we conducted exploratory analyses to examine if there were differences in VE by time since vaccination and whether cases had presumptive evidence of active follow-up consistent with them qualifying as truly "confirmed" according to case definitions (as follow-up may have been incomplete; see "Methods"). We found no differences in ORs by time since vaccine receipt, but found that cases with no evidence of active follow-up were 5 times more likely to have been vaccinated $(40.4 \%$; 23 of 57$)$ than those with evidence suggesting follow-up (9.1\%; 9 of 99), resulting in substantially different ORs and VE estimates (Table A3).

\section{DISCUSSION}

Among older adults (mean age, 61 years), we found significant effectiveness of acellular pertussis vaccine against PCRconfirmed pertussis infection of approximately $50 \%$. We did not find evidence of waning protection with increasing age or with time since vaccination, but these subanalyses lacked statistical power.

Our findings add substantially to current evidence on acellular pertussis VE in older adults. The only randomized trial estimating efficacy of the adult formulation of acellular pertussis vaccine had participants with a mean age of 35 years, excluded adults aged over 65 , and had limited statistical power [8]. Of 11 other published studies estimating VE [22-30], all but one [11] included only adolescents, most of whom were primed with acellular vaccines. The earlier case-control study estimating VE in older adults was based on PCR-confirmed cases in a US health maintenance organization database. They found, in data restricted to cases born in the prevaccine era (before 1950; mean age, 69 years; case $\mathrm{N}=61$ ), that $\mathrm{VE}$ was low and nonsignificant (24\%; 95\% CI, -59-64\%), but among those born in the wholecell vaccine era (1950-1985; mean age, 43 years; case $\mathrm{N}=129$ ) VE was substantially higher (68\%; 95\% CI, 46-82\%) [11]. Our findings contrast, as we found no significant differences in VE by age ( $<65$ vs $\geq 65$ years) nor by equivalent birth cohorts (born before 1950 vs 1950 or later).

One major methodological difference between the US study and ours is that we had population-based controls while the US study had both PCR-negative and population-based controls. While the US study found that the use of PCR-negative controls resulted in lower VE estimates overall, this difference was only observed among the younger subgroup of participants (mean age, 11 years), who had all been exposed to acellular vaccines, but not in the 2 older subgroups, suggesting this potential for 
Table 2. Characteristics of Cases and Controls in Polymerase Chain Reaction-Analysis Population

\begin{tabular}{|c|c|c|c|}
\hline & $\begin{array}{l}\text { Cases, } \\
\text { N (\%) }\end{array}$ & $\begin{array}{l}\text { Controls, } \\
\text { N (\%) }\end{array}$ & $P$ \\
\hline Total & 172 (39.3) & $266(60.7)$ & \\
\hline \multicolumn{4}{|l|}{ Recruitment date } \\
\hline Before 1 July 2007 & $24(14.0)$ & $36(13.5)$ & .99 \\
\hline 1 July-31 December 2007 & $13(7.6)$ & $21(7.9)$ & \\
\hline 1 January-30 June 2008 & $44(25.6)$ & $67(25.2)$ & \\
\hline After 30 June 2008 & $91(52.9)$ & $142(53.4)$ & \\
\hline Mean (SD) age at diagnosis/index, years & $61.1(7.7)$ & $61.3(7.4)$ & .75 \\
\hline \multicolumn{4}{|l|}{ Age at diagnosis/index date (years) } \\
\hline $45-49$ & $11(6.4)$ & $19(7.1)$ & .35 \\
\hline $50-54$ & $37(21.5)$ & $43(16.2)$ & \\
\hline $55-59$ & $31(18.0)$ & 45 (16.9) & \\
\hline $60-64$ & $40(23.3)$ & $83(31.2)$ & \\
\hline $65-69$ & $29(16.9)$ & $49(18.4)$ & \\
\hline$\geq 70$ & $24(14.0)$ & $27(10.2)$ & \\
\hline \multicolumn{4}{|l|}{ Sex } \\
\hline Male & $54(31.4)$ & $83(31.2)$ & .97 \\
\hline Female & $118(68.6)$ & $183(68.8)$ & \\
\hline \multicolumn{4}{|l|}{ Body mass index $\left(\mathrm{kg} / \mathrm{m}^{2}\right)$} \\
\hline$<25$ & 60 (34.9) & $97(36.5)$ & .58 \\
\hline $25-30$ & $66(38.4)$ & 87 (32.7) & \\
\hline$\geq 30$ & $29(16.9)$ & $56(21.1)$ & \\
\hline Unknown & $17(9.9)$ & $26(9.8)$ & \\
\hline \multicolumn{4}{|l|}{ Highest educational level } \\
\hline Certificate or lower & $111(64.5)$ & $171(64.3)$ & .96 \\
\hline University or higher & $56(32.6)$ & $86(32.3)$ & \\
\hline Unknown & $5(2.9)$ & $9(3.4)$ & \\
\hline \multicolumn{4}{|l|}{ Residence } \\
\hline Major city & $88(51.2)$ & $121(45.5)$ & .11 \\
\hline Inner regional & $69(40.1)$ & $102(38.3)$ & \\
\hline $\begin{array}{l}\text { Outer regional or remote } \\
\text { or very remote }\end{array}$ & $11(6.4)$ & $36(13.5)$ & \\
\hline Unknown & $4(2.3)$ & 7 (2.6) & \\
\hline \multicolumn{4}{|l|}{ Annual household income (AUD) } \\
\hline$<70000$ & $74(43.0)$ & $129(48.5)$ & .44 \\
\hline$\geq 70000$ & $89(51.7)$ & $121(45.5)$ & \\
\hline Unknown & $9(5.2)$ & $16(6.0)$ & \\
\hline \multicolumn{4}{|l|}{ Smoking status } \\
\hline Never & $106(61.6)$ & $157(59.0)$ & .19 \\
\hline Past & $58(33.7)$ & $86(32.3)$ & \\
\hline Current & $3(1.7)$ & $16(6.0)$ & \\
\hline Unknown & $5(2.9)$ & $7(2.6)$ & \\
\hline \multicolumn{4}{|l|}{ Asthma } \\
\hline No & $144(83.7)$ & $222(83.5)$ & .99 \\
\hline Yes & $24(14.0)$ & 37 (13.9) & \\
\hline Unknown & $4(2.3)$ & $7(2.6)$ & \\
\hline \multicolumn{4}{|l|}{ Contact with children } \\
\hline No & $92(53.5)$ & $124(46.6)$ & $<.001$ \\
\hline Yes & $63(36.6)$ & $67(25.2)$ & \\
\hline Unknown & $17(9.9)$ & $75(28.2)$ & \\
\hline \multicolumn{4}{|l|}{ Attended cancer screening program } \\
\hline No & $20(11.6)$ & $22(8.3)$ & .24 \\
\hline Yes & $146(84.9)$ & $235(88.3)$ & \\
\hline Unknown & $6(3.5)$ & $9(3.4)$ & \\
\hline
\end{tabular}

Percentages are column percentages. Abbreviation: AUD, Australian dollars. bias may not be uniform across age groups. Differences in VE between test-negative and population-based controls are presumably due to test-negative controls being more similar to the cases (ie, having more risk factors leading to pertussis testing and subsequent diagnosis than population-based controls). In our analyses, to account for differences in the propensity for pertussis testing and diagnosis, we adjusted for characteristics including contact with children, comorbidities (asthma, smoking, $\mathrm{BMI}$ ), and healthcare seeking (attending cancer screening). This adjustment led to an approximately $10 \%$ increase in the estimated VE rather than any decrease, and was primarily driven by adjustment for contact with children. The other factors did not materially change our estimates, suggesting that they were not major confounders of our results.

None of the earlier reports of VE/efficacy in older adults [8, 11] attempted to examine waning. Cost-effectiveness analyses of vaccination of older adults with acellular pertussis vaccine have assumed mean durations of protection of 8 years [31] based mostly on studies in children [32], although a much shorter duration of protection has been reported among those who received all primary doses as acellular vaccine in childhood [12]. While we had limited statistical power, we found that up to 6 years after vaccination, the point estimate for VE showed no declining trend. Based on their age, most adults in our study would have been primed, either by having pertussis infection or having been vaccinated with whole-cell vaccines as a child. Therefore, our findings regarding both the effectiveness and duration of protection must be interpreted in this context.

The finding of an association (although not significant) between pertussis vaccination and a pertussis notification on the basis of positive single-titer serology was surprising and inconsistent with our results for PCR-confirmed cases. In Australia, pertussis notification using single high-titer serology requires both clinical evidence and exclusion of recent vaccination (see Appendix). Potential flaws in this case reporting include, first, variations in the sensitivity and specificity of serological tests used by referring laboratories and, second, that public health unit workload may preclude active follow-up of notifications made directly from laboratories, which account for most pertussis notifications in NSW [33]. Our post hoc analyses comparing serology cases with and without proxy evidence of active follow-up (Table A3) support the likelihood of substantial misclassification among cases identified solely through serologic diagnosis. These findings have implications for pertussis surveillance in Australia, particularly in adults, a population for whom, historically, the majority of notifications were based on serological confirmation [2]. Indeed, as more adults are vaccinated as part of population-wide programs targeting pregnant women or older adults [13], diagnoses based primarily on pertussis serology may become more unreliable and 


\begin{tabular}{|c|c|c|c|c|c|c|c|}
\hline & $\begin{array}{c}\text { Cases } \\
\mathbf{N}\end{array}$ & $\begin{array}{c}\text { Controls* } \\
\mathbf{N}\end{array}$ & $\begin{array}{l}\text { Unadjusted } \\
\text { OR }\end{array}$ & $\begin{array}{l}\text { Adjusted+ } \\
\text { OR }\end{array}$ & $\begin{array}{c}\text { Adjusted+ } \\
\text { vaccine effectiveness (\%) } \\
(95 \% \mathrm{Cl})\end{array}$ & $P$ value & \\
\hline \multicolumn{8}{|c|}{ 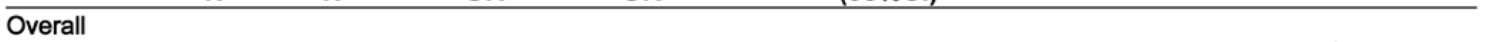 } \\
\hline Unvaccinated & 280 & 424 & Ref & Ref & Ref & & \\
\hline Vaccinated & 53 & 82 & 0.98 & 0.92 & $8(-36$ to 37$)$ & 0.69 & $=$ \\
\hline \multicolumn{8}{|l|}{ By case type } \\
\hline \multicolumn{8}{|l|}{ PCR } \\
\hline Unvaccinated & 152 & 214 & Ref & Ref & Ref & & \\
\hline Vaccinated & 20 & 52 & 0.54 & 0.48 & 52 (15 to 73 ) & 0.01 & $\longrightarrow-$ \\
\hline \multicolumn{8}{|l|}{ Serology } \\
\hline Unvaccinated & 124 & 202 & Ref & Ref & Ref & & \\
\hline \multirow[t]{2}{*}{ Vaccinated } & 32 & 30 & 1.74 & 1.55 & $-55(-177$ to 13$)$ & 0.14 & $\longleftrightarrow$ \\
\hline & & & & & & & $\Gamma_{1}$ \\
\hline * Matched on & recrui & t date, & and sex & & & & 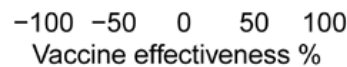 \\
\hline +Adjusted for & contact & with childre & en and region & of residence & & & $\begin{array}{l}\text { Vaccine eftectiveness \% } \\
(95 \% \mathrm{Cl})\end{array}$ \\
\hline
\end{tabular}

Figure 2. Odds ratios and vaccine effectiveness of acellular pertussis vaccine, prespecified population ( $\mathrm{N}=839$ ). Abbreviations: $\mathrm{Cl}$, confidence interval; $\mathrm{OR}$, odds ratio; $\mathrm{PCR}$, polymerase chain reaction; Ref, reference.

routine surveillance systems will need to adapt to maintain case specificity.

Our study strengths include the relatively large sample size and nested matched design, which allowed us to select controls who were similar to cases in terms of not only age and sex but also characteristics such as health-seeking behavior, which has been shown to be higher overall in this cohort than in the general population [15]. We were also able to adjust our analyses for underlying factors that may be associated with pertussis incidence and severity $[4,6]$, as well as characteristics associated with greater likelihood to participate in the study. Limitations include that our response rate was less than anticipated, which meant that we had insufficient statistical power for robust VE estimates in subgroups including in those aged 65 years and older and according to time since vaccination. Also, response rates differed by case and control status and may bias our VE estimates away from the null if responding controls were more likely to be vaccinated, although this was not the case in the main prespecified analysis population (see Figure 2). As mentioned, we also lacked PCR-negative controls, with the majority of the controls' health practitioners responding that they had not been tested for pertussis. Finally, we lacked data on patient symptoms, which would have assisted in interpretation of the findings based on serological diagnoses.

Overall, we found in adults aged, on average, 61 years that acellular pertussis vaccine is approximately $50 \%$ effective in preventing PCR-confirmed pertussis infection. Together with findings from other studies $[8,11]$, we conclude that while

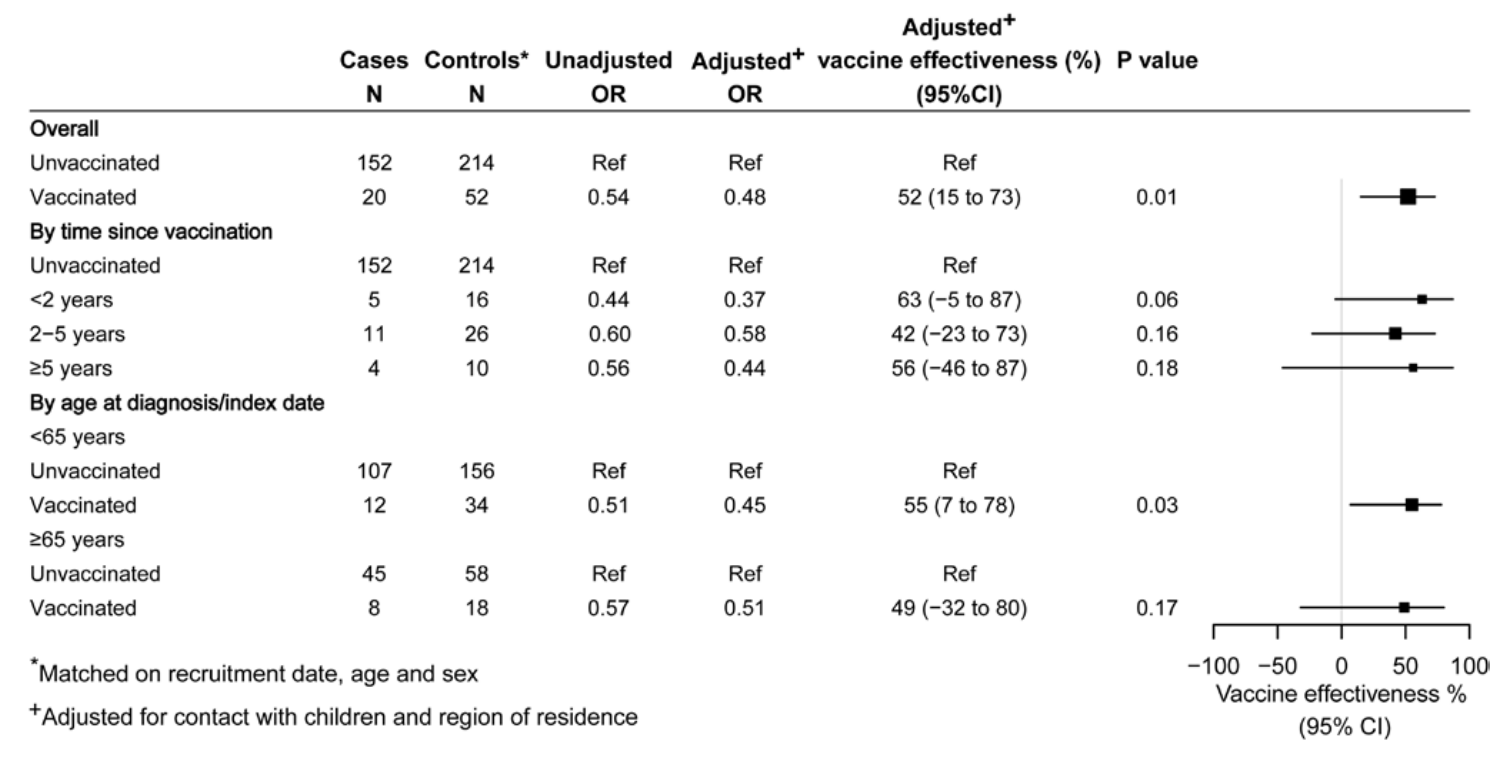

Figure 3. Odds ratios and vaccine effectiveness of acellular pertussis vaccine, $\mathrm{PCR}$-only population $(\mathrm{N}=438)$. Cl, confidence interval; $\mathrm{OR}$, odds ratio; $\mathrm{PCR}$, polymerase chain reaction; Ref, reference. 
effectiveness of the adult acellular pertussis vaccine may decline in older age, it still confers a level of protection that is comparable to that often reported for vaccines such as influenza [34], which are routinely recommended for older adults. While a more effective vaccine would be ideal, Tdap should still be considered an important intervention among public health strategies to prevent infectious respiratory morbidity in older adults.

\section{Notes}

Acknowledgments. The 45 and Up Study is managed by the Sax Institute in collaboration with major partner Cancer Council NSW and the following partners: the National Heart Foundation of Australia (NSW Division); NSW Ministry of Health; NSW Government Family and Community Services-Ageing, Carers and the Disability Council; and the Australian Red Cross Blood Service. The authors thank the many thousands of people participating in the 45 and Up Study and the Centre for Health Record Linkage and NSW Ministry of Health for providing the linked notification and Admitted Patient Data Collection and Register of Births Deaths and Marriages data. The authors thank Bruce Armstrong and Paula Spokes for their advice on study design, analysis, and interpretation.

Financial support. This project was supported by the Australian National Health and Medical Research Council (NHMRC) (grant number 1107008). B. C. L. and C. R. M. are supported by NHMRC fellowships.

Potential conflicts of interest. B. C. L. and A. H. hold grants from the Australian National Health and Medical Research Council during the conduct of the study. B. C. L. has held shares in CSL Limited and served once on an advisory board for Sanofi-Pasteur. All other authors report no potential conflicts. All authors have submitted the ICMJE Form for Disclosure of Potential Conflicts of Interest. Conflicts that the editors consider relevant to the content of the manuscript have been disclosed.

\section{References}

1. Cherry JD. Epidemic pertussis in 2012-the resurgence of a vaccine-preventable disease. N Engl J Med 2012; 367:785-7.

2. Pillsbury A, Quinn HE, McIntyre PB. Australian vaccine preventable disease epidemiological review series: pertussis, 2006-2012. Commun Dis Intell Q Rep 2014 38:E179-94.

3. Liang JL, Tiwari T, Moro P, et al. Prevention of pertussis, tetanus, and diphtheria with vaccines in the United States: recommendations of the Advisory Committee on Immunization Practices (ACIP). MMWR Recomm Rep 2018; 67:1-44.

4. De Serres G, Shadmani R, Duval B, et al. Morbidity of pertussis in adolescents and adults. J Infect Dis 2000; 182:174-9.

5. Thomas PF, McIntyre PB, Jalaludin BB. Survey of pertussis morbidity in adults in western Sydney. Med J Aust 2000; 173:74-6.

6. Liu BC, McIntyre P, Kaldor JM, Quinn HE, Ridda I, Banks E. Pertussis in older adults: prospective study of risk factors and morbidity. Clin Infect Dis 2012 55:1450-6

7. Cortese MM, Baughman AL, Brown K, Srivastava P. A "new age" in pertussis prevention new opportunities through adult vaccination. Am J Prev Med 2007; 32:177-85.

8. Ward JI, Cherry JD, Chang SJ, et al; APERT Study Group. Efficacy of an acellular pertussis vaccine among adolescents and adults. N Engl J Med 2005; 353:1555-63.

9. Van Damme P, McIntyre P, Grimprel E, et al. Immunogenicity of the reducedantigen-content dTpa vaccine $\left(\operatorname{Boostrix}\left({ }^{\circ}\right)\right)$ in adults 55 years of age and over: a sub-analysis of four trials. Vaccine 2011; 29:5932-9.

10. Weston WM, Friedland LR, Wu X, Howe B. Vaccination of adults 65 years of age and older with tetanus toxoid, reduced diphtheria toxoid and acellular pertussis vaccine (Boostrix $\left(^{\oplus}\right)$ ): results of two randomized trials. Vaccine 2012; 30:1721-8.

11. Baxter R, Bartlett J, Rowhani-Rahbar A, Fireman B, Klein NP. Effectiveness of pertussis vaccines for adolescents and adults: case-control study. BMJ 2013; 347:f4249.

12. Klein NP, Bartlett J, Rowhani-Rahbar A, Fireman B, Baxter R. Waning protection after fifth dose of acellular pertussis vaccine in children. N Engl J Med 2012; 367:1012-9.
13. Dyda A, McIntyre P, Karki S, et al. Pertussis vaccination in a cohort of older Australian adults following a cocooning vaccination program. Vaccine $\mathbf{2 0 1 8}$ 36:4157-60.

14. Australian Immunisation Handbook. 2019. Available at: https:// immunisationhandbook.health.gov.au/recommendations? $\%$ 5B0\%5D =field related_diseases\%3A3701. Accessed February 2019.

15. 45 and Up Study Collaborators; Banks E, Redman S, et al. Cohort profile: the 45 and Up Study. Int J Epidemiol 2008; 37:941-7.

16. Title NSW Centre for Health Record Linkage. Available at: http://www.cherel.org au/. Accessed February 2019.

17. New South Wales Government. Public Health Act 2010. Available at: https:// www.legislation.nsw.gov.au/\#/view/act/2010/127. Accessed February 2019.

18. Australian Government Department of Health. Australian national notifiable diseases and case definitions. Available at: http://www.health.gov.au/internet/main/ publishing.nsf/Content/cda-surveil-nndss-casedefs-cd_pertus.htm. Accessed February 2019

19. Innes K, Peasley K, Roberts R. Ten down under: implementing ICD-10 in Australia. J AHIMA 2000; 71:52-6.

20. Halperin BA, Morris A, Mackinnon-Cameron D, et al. Kinetics of the antibody response to tetanus-diphtheria-acellular pertussis vaccine in women of childbearing age and postpartum women. Clin Infect Dis 2011; 53:885-92.

21. The Comprehensive R Archive Network. Available at: https://cranr-projectorg/. Accessed April 2019.

22. Koepke R, Eickhoff JC, Ayele RA, et al. Estimating the effectiveness of tetanusdiphtheria-acellular pertussis vaccine (Tdap) for preventing pertussis: evidence of rapidly waning immunity and difference in effectiveness by Tdap brand. J Infect Dis 2014; 210:942-53.

23. Klein NP, Bartlett J, Fireman B, Baxter R. Waning Tdap effectiveness in adolescents. Pediatrics 2016; 137:e20153326.

24. Acosta AM, DeBolt C, Tasslimi A, et al. Tdap vaccine effectiveness in adolescents during the 2012 Washington State pertussis epidemic. Pediatrics 2015; 135:981-9.

25. Breakwell L, Kelso P, Finley C, et al. Pertussis vaccine effectiveness in the setting of pertactin-deficient pertussis. Pediatrics 2016; 137:05.

26. Wei SC, Tatti K, Cushing K, et al. Effectiveness of adolescent and adult tetanus, reduced-dose diphtheria, and acellular pertussis vaccine against pertussis. Clin Infect Dis 2010; 51:315-21.

27. Rank C, Quinn HE, McIntyre PB. Pertussis vaccine effectiveness after mass immunization of high school students in Australia. Pediatr Infect Dis J 2009; 28:152-3.

28. Haller S, Dehnert M, Karagiannis I, et al. Effectiveness of routine and booster pertussis vaccination in children and adolescents, federal state of Brandenburg, Germany, 2002-2012. Pediatr Infect Dis J 2015; 34:513-9.

29. Terranella A, Rea V, Griffith M, et al. Vaccine effectiveness of tetanus toxoid, reduced diphtheria toxoid, and acellular pertussis vaccine during a pertussis outbreak in Maine. Vaccine 2016; 34:2496-500.

30. Liko J, Robison SG, Cieslak PR. Pertussis vaccine performance in an epidemic year-Oregon, 2012. Clin Infect Dis 2014; 59:261-3.

31. McGarry LJ, Krishnarajah G, Hill G, et al. Cost-effectiveness analysis of Tdap in the prevention of pertussis in the elderly. PLoS One 2013; 8:e67260.

32. Wendelboe AM, Van Rie A, Salmaso S, Englund JA. Duration of immunity against pertussis after natural infection or vaccination. Pediatr Infect Dis J 2005 24:S58-61.

33. Allen CJ, Ferson MJ. Notification of infectious diseases by general practitioners: quantitative and qualitative study. Med J Aust 2000; 172:325-8.

34. Belongia EA, Simpson MD, King JP, et al. Variable influenza vaccine effectivenes by subtype: a systematic review and meta-analysis of test-negative design studies. Lancet Infect Dis 2016; 16:942-51.

\section{APPENDIX: NATIONAL NOTIFIABLE DISEASES CASE DEFINITION FOR PERTUSSIS REPORTING}

Both confirmed cases and probable cases should be notified.

Confirmed case: A confirmed case requires either laboratory definitive evidence OR laboratory suggestive evidence AND clinical evidence.

Probable case: A probable case requires clinical evidence AND epidemiological evidence. 
Laboratory definitive evidence: Isolation of Bordetella pertussis OR detection of $B$. pertussis by nucleic acid testing OR seroconversion in paired sera for $B$. pertussis using whole-cell or specific $B$. pertussis antigen(s) in the absence of recent pertussis vaccination.

Laboratory suggestive evidence: In the absence of recent vaccination, significant change (increase or decrease) in antibody level (IgG, IgA) to B. pertussis whole-cell or B. pertussisspecific antigen(s) OR single high IgG and/or IgA titer to pertussis toxin (PT) OR single high IgA titer to whole-cell B. pertussis antigen.

Clinical evidence: A coughing illness lasting 2 or more weeks
OR paroxysms of coughing OR inspiratory whoop OR posttussive vomiting.

Epidemiological evidence: An epidemiological link is established when there is contact between 2 people involving a plausible mode of transmission at a time when one of them is likely to be infectious (from the catarrhal stage, approximately 1 week before, to 3 weeks after onset of cough) AND the other has an illness that starts within 6 to 20 days after this contact AND at least 1 case in the chain of epidemiologically linked cases (which may involve many cases) is a confirmed case with either laboratory definitive or laboratory suggestive evidence.

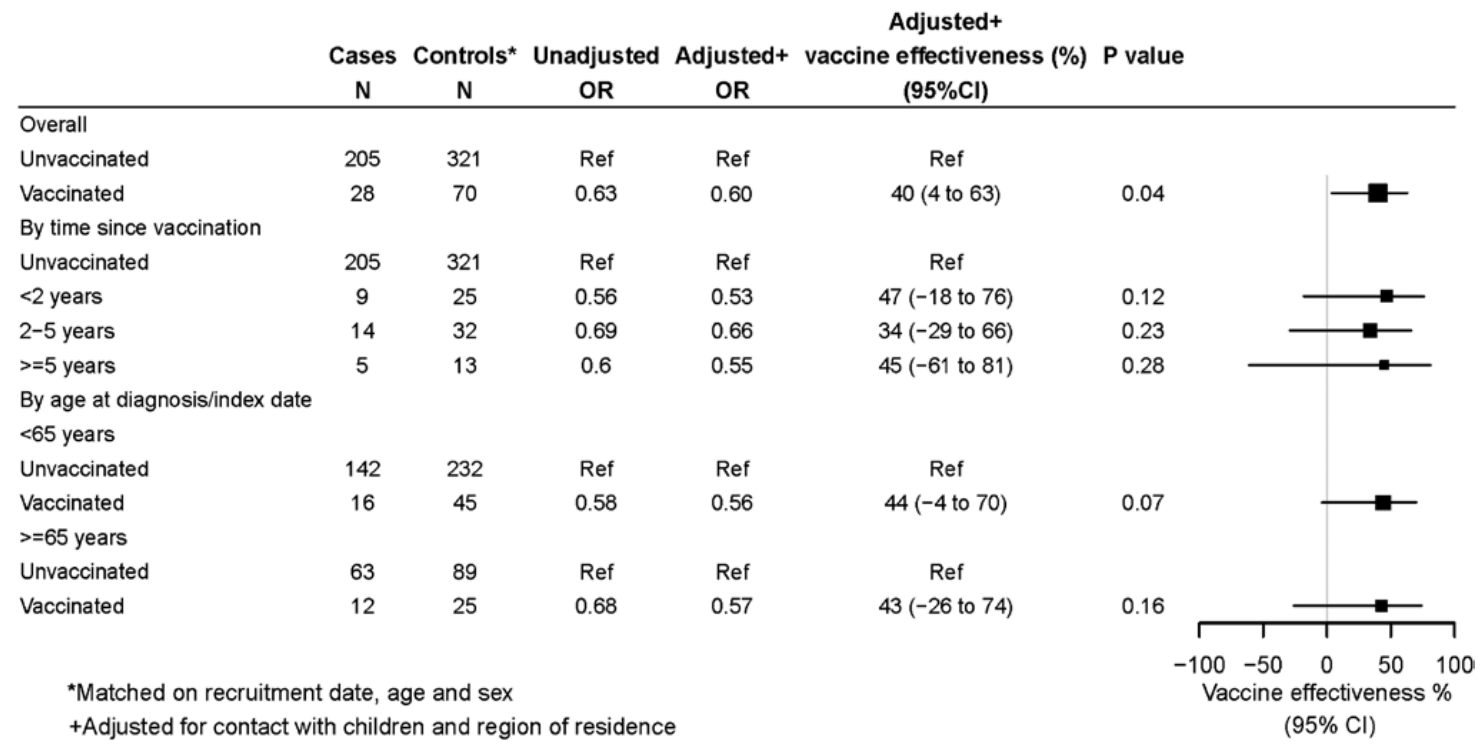

Appendix Figure A1. Odds ratios and vaccine effectiveness of acellular pertussis vaccine: PCR-only population, new matched controls ( $N=624)$. Abbreviations: $\mathrm{Cl}$, confidence interval; OR, odds ratio; Ref, reference. 
Table A1. Number and Percentage of Participants Responding Overall and According to Case and Control Status

\begin{tabular}{|c|c|c|c|}
\hline & Cases, n/N (\% Responded) & Controls, n/N (\% Responded) & Total, n/N (\% Responded) \\
\hline Total & $671 / 1112$ (60.3) & 1610/3291 (48.9) & $2281 / 4403$ (51.8) \\
\hline \multicolumn{4}{|c|}{ Age at diagnosis/index date (years) } \\
\hline $45-64$ & $441 / 663(66.5)$ & $1056 / 1984(53.2)$ & $1497 / 2647(56.6)$ \\
\hline$\geq 65$ & $230 / 449(51.2)$ & $554 / 1307$ (42.4) & $784 / 1756$ (44.6) \\
\hline \multicolumn{4}{|l|}{ Sex } \\
\hline Male & 215/395 (54.4) & $546 / 1184(46.1)$ & $761 / 1579$ (48.2) \\
\hline Female & $456 / 717(63.6)$ & $1064 / 2107$ (50.5) & $1520 / 2824(53.8)$ \\
\hline \multicolumn{4}{|l|}{ Residence } \\
\hline Major city & $280 / 503(55.7)$ & $797 / 1698$ (46.9) & $1077 / 2201$ (48.9) \\
\hline Regional or remote & $365 / 576(63.4)$ & $778 / 1534(50.7)$ & $1143 / 2110(54.2)$ \\
\hline Unknown & 26/33 (78.8) & $35 / 59(59.3)$ & $61 / 92(66.3)$ \\
\hline \multicolumn{4}{|c|}{ Annual household income (AUD) } \\
\hline$<70000$ & $339 / 596(56.9)$ & $825 / 1730(47.7)$ & $1164 / 2326(50.0)$ \\
\hline$\geq 70000$ & $311 / 465(66.9)$ & 734/1413 (51.9) & 1045/1878 (55.6) \\
\hline Unknown & $21 / 51(41.2)$ & $51 / 148(34.5)$ & $72 / 199$ (36.2) \\
\hline \multicolumn{4}{|c|}{ Highest educational level } \\
\hline No university & $477 / 815(58.5)$ & $1124 / 2436(46.1)$ & $1601 / 3251(49.2)$ \\
\hline University & $186 / 278(66.9)$ & $474 / 821(57.7)$ & $660 / 1099(60.1)$ \\
\hline Unknown & $8 / 19(42.1)$ & $12 / 34(35.3)$ & $20 / 53(37.7)$ \\
\hline \multicolumn{4}{|l|}{ Smoking status } \\
\hline Never & $417 / 665(62.7)$ & $968 / 1931(50.1)$ & $1385 / 2596(53.4)$ \\
\hline Past & $220 / 380(57.9)$ & $543 / 1107(49.1)$ & $763 / 1487(51.3)$ \\
\hline Current & $34 / 67(50.7)$ & $98 / 252$ (38.9) & $132 / 319(41.4)$ \\
\hline Unknown & & $1 / 1(100.0)$ & $1 / 1(100.0)$ \\
\hline \multicolumn{4}{|c|}{ Attended cancer screening program } \\
\hline No & $73 / 121(60.3)$ & $174 / 410(42.4)$ & $247 / 531(46.5)$ \\
\hline Yes & $560 / 933(60.0)$ & $1381 / 2763(50.0)$ & $1941 / 3696$ (52.5) \\
\hline Unknown & $38 / 58(65.5)$ & $55 / 118(46.6)$ & $93 / 176(52.8)$ \\
\hline \multicolumn{4}{|l|}{ Case diagnosis method } \\
\hline PCR & $332 / 528$ (62.9) & $\ldots$ & $\ldots$ \\
\hline Serology & $330 / 565(58.4)$ & $\cdots$ & $\cdots$ \\
\hline Unknown & $9 / 19(47.4)$ & $\cdots$ & $\cdots$ \\
\hline \multicolumn{4}{|l|}{ Diagnosis year } \\
\hline$<2009$ & $96 / 167(57.5)$ & $\ldots$ & $\ldots$ \\
\hline 2009 & $135 / 226(59.7)$ & $\cdots$ & $\ldots$ \\
\hline 2010 & 89/153 (58.2) & $\ldots$ & $\ldots$ \\
\hline 2011 & $107 / 180(59.4)$ & $\ldots$ & $\ldots$ \\
\hline 2012 & $66 / 110(60.0)$ & $\cdots$ & $\cdots$ \\
\hline 2013 & $41 / 61(67.2)$ & $\cdots$ & $\ldots$ \\
\hline 2014 & 48/71 (67.6) & $\ldots$ & $\ldots$ \\
\hline 2015 & $89 / 135$ (65.9) & $\ldots$ & $\ldots$ \\
\hline
\end{tabular}

Abbreviations: AUD, Australian dollars; $\mathrm{PCR}$, polymerase chain reaction. 
Table A2. Number and Percentage of Responding Participants With Validated Vaccination Data From Their Health Care Provider Overall and According to Case and Control Status

\begin{tabular}{|c|c|c|c|}
\hline & Cases, n/N (\% Responded) & Controls, n/N (\% Responded) & Total, n/N (\% Responded) \\
\hline Total & $463 / 671(69.0)$ & $1080 / 1610(67.1)$ & $1543 / 2281(67.6)$ \\
\hline \multicolumn{4}{|c|}{ Age at diagnosis/index date (years) } \\
\hline $45-64$ & $294 / 441(66.7)$ & $707 / 1056$ (67) & $1001 / 1497(66.9)$ \\
\hline$\geq 65$ & $169 / 230(73.5)$ & $373 / 554(67.3)$ & $542 / 784(69.1)$ \\
\hline \multicolumn{4}{|l|}{ Sex } \\
\hline Male & $148 / 215(68.8)$ & $348 / 546(63.7)$ & 496/761 (65.2) \\
\hline Female & $315 / 456(69.1)$ & $732 / 1064(68.8)$ & $1047 / 1520(68.9)$ \\
\hline \multicolumn{4}{|l|}{ Residence } \\
\hline Major city & $201 / 280(71.8)$ & $525 / 797$ (65.9) & $726 / 1077(67.4)$ \\
\hline Regional or remote & $243 / 365(66.6)$ & $530 / 778(68.1)$ & $773 / 1143(67.6)$ \\
\hline Unknown & $19 / 26(73.1)$ & $25 / 35(71.4)$ & $44 / 61(72.1)$ \\
\hline \multicolumn{4}{|c|}{ Annual household income (AUD) } \\
\hline$<70000$ & $236 / 339(69.6)$ & $545 / 825(66.1)$ & $781 / 1164(67.1)$ \\
\hline$\geq 70000$ & $215 / 311(69.1)$ & 497/734 (67.7) & $712 / 1045(68.1)$ \\
\hline Unknown & $12 / 21(57.1)$ & $38 / 51(74.5)$ & $50 / 72(69.4)$ \\
\hline \multicolumn{4}{|c|}{ Highest educational level } \\
\hline No university & $329 / 477(69.0)$ & $736 / 1124(65.5)$ & $1065 / 1601(66.5)$ \\
\hline University & 130/186 (69.9) & $337 / 474(71.1)$ & $467 / 660(70.8)$ \\
\hline Unknown & $4 / 8(50.0)$ & $7 / 12(58.3)$ & $11 / 20(55.0)$ \\
\hline \multicolumn{4}{|l|}{ Smoking status } \\
\hline Never & $305 / 417(73.1)$ & $665 / 968(68.7)$ & $970 / 1385(70.0)$ \\
\hline Past & $144 / 220(65.5)$ & $349 / 543(64.3)$ & $493 / 763(64.6)$ \\
\hline Current & $14 / 34(41.2)$ & $65 / 98(66.3)$ & 79/132 (59.8) \\
\hline Unknown & & $1 / 1(100.0)$ & $1 / 1(100.0)$ \\
\hline \multicolumn{4}{|c|}{ Attended cancer screening program } \\
\hline No & $53 / 73(72.6)$ & 103/174 (59.2) & $156 / 247(63.2)$ \\
\hline Yes & $385 / 560(68.8)$ & $940 / 1381(68.1)$ & 1325/1941 (68.3) \\
\hline Unknown & $25 / 38(65.8)$ & $37 / 55(67.3)$ & $62 / 93(66.7)$ \\
\hline \multicolumn{4}{|l|}{ Case diagnosis method } \\
\hline PCR & 233/332 (70.2) & $\ldots$ & $\ldots$ \\
\hline Serology & $223 / 330(67.6)$ & $\ldots$ & \\
\hline Unknown & $7 / 9(77.8)$ & $\ldots$ & $\ldots$ \\
\hline \multicolumn{4}{|l|}{ Diagnosis year } \\
\hline$<2009$ & 69/96 (71.9) & $\ldots$ & $\ldots$ \\
\hline 2009 & $83 / 135(61.5)$ & $\ldots$ & $\ldots$ \\
\hline 2010 & $64 / 89(71.9)$ & $\ldots$ & $\ldots$ \\
\hline 2011 & 78/107 (72.9) & $\ldots$ & $\ldots$ \\
\hline 2012 & 46/66 (69.7) & $\ldots$ & $\ldots$ \\
\hline 2013 & $30 / 41(73.2)$ & $\ldots$ & $\ldots$ \\
\hline 2014 & $30 / 48(62.5)$ & $\ldots$ & $\ldots$ \\
\hline 2015 & $63 / 89$ (70.8) & $\ldots$ & $\ldots$ \\
\hline
\end{tabular}

Abbreviations: AUD, Australian dollars; PCR, polymerase chain reaction. 
Table A3. Odds Ratios and Vaccine Effectiveness of Acellular Pertussis Vaccine, Serology-only Population ( $\mathrm{N}=388$ ) Overall, by Time Since Vaccination, and According to Whether There Was a Record of Active Follow-up

\begin{tabular}{|c|c|c|c|c|c|c|}
\hline & Cases & Controls $^{a}$ & Unadjusted OR & Adjusted OR ${ }^{b}$ & Adjusted VE, \% (95\% Cl) & $P$ \\
\hline \multicolumn{7}{|l|}{ Serology subgroup } \\
\hline Unvaccinated & 124 & 202 & Ref & Ref & Ref & \\
\hline Vaccinated & 32 & 30 & 1.74 & 1.55 & $-55(-177-13)$ & .14 \\
\hline \multicolumn{7}{|l|}{ By time since vaccination } \\
\hline Unvaccinated & 124 & 202 & Ref & Ref & Ref & \\
\hline Vaccinated $\leq 1$ year prior & 6 & 9 & 1.09 & 1.27 & $-27(-281-60)$ & .68 \\
\hline Vaccinated $>1$ year prior & 26 & 21 & 2.02 & 1.72 & $-72(-241-12)$ & .11 \\
\hline \multicolumn{7}{|l|}{ Follow-up record } \\
\hline Unvaccinated & 90 & 132 & Ref & Ref & Ref & \\
\hline Vaccinated & 9 & 13 & 1.02 & 0.83 & $17(-128-69)$ & .72 \\
\hline \multicolumn{7}{|l|}{ No follow-up record } \\
\hline Unvaccinated & 34 & 70 & Ref & Ref & Ref & \\
\hline Vaccinated & 23 & 17 & 2.79 & 2.57 & $-157(-464$ to -17$)$ & .02 \\
\hline
\end{tabular}

Serology cases were classified as having no record of active follow-up by the Public Health Unit if their notification record had missing data in the fields requesting information on hospitalization or death. Both are data fields that cannot be obtained through the routine laboratory notification. Abbreviations: $\mathrm{Cl}$, confidence interval; OR, odds ratio; Ref, reference.

${ }^{a}$ Matched on recruitment date, age, and sex.

${ }^{\mathrm{b}}$ Adjusted for contact with children and region of residence. 